\title{
Causes and assessment of faecal incontinence
}

\section{Sonya Chelvanayagam and, Christine Norton}

\begin{abstract}
Faecal incontinence remains a taboo subject and patients frequently conceal their symptoms, because of fear and embarrassment. Sensitive and appropriate assessment will encourage people to seek advice and treatment if required. This article discusses the causes and assessment of faecal incontinence. It explains the structure and function of the anal sphincters in maintaining continence. Assessment of bowel control is discussed in depth. Questions are suggested which will help to identify problems and thereby enable access to appropriate care.

\section{Introduction}

Faecal incontinence is defined as the involuntary or inappropriate passing of liquid or solid stool (Royal College of Physicians, 1995). Anal incontinence is the term used to describe any involuntary leakage from the anus, whether of solid, liquid or flatus.

Faecal incontinence is estimated to affect over $1 \%$ of the adult population (Nelson et al, 1995). However, this may be an underestimate, given that the symptom is viewed as socially unacceptable and probably only one third of people with faecal incontinence seek professional help (Nelson et al, 1995). Even individuals seen by bowel specialists do not voluntarily divulge that they have faecal incontinence (Leigh and Turnberg, 1982; Johanson and Lafferty, 1996)

Community nurses and midwives, who are involved with the patient in his/her own environment and have an established rapport, are ideally placed to identify a patient's compromised continence and initiate or refer the patient for appropriate investigations and management
\end{abstract}

\section{Causes}

\section{Anal sphincter damage or weakness}

Bowel continence is maintained primarily by two cylindrical anal sphincter muscles (Figure 1):

- The internal anal sphincter is a thin (2-3mm in diameter) smooth muscle which remains continuously contracted (unless opening the bowels) and therefore maintains closure of the anal canal. An intact internal sphincter prevents passive seepage of stool and flatus.

- The external anal sphincter is composed of voluntary (striated) muscle. It is this muscle that is squeezed and contracted when deferring defecation following an urge to empty the bowel. 
Damage to one or both sphincters is the most common cause of faecal incontinence in younger adults and is often caused by obstetric trauma. Sultan et al (1993) found that one-third of women in their study sustained some degree of anal sphincter damage during a first vaginal delivery and over $80 \%$ of those having a forceps-assisted delivery had sphincter damage; in contrast, none of the women delivering by caesarean section sustained any changes in sphincter anatomy or function.

Obstetric tears, particularly third-degree tears extending from the vagina through to the anal sphincter, can also result in incontinence, often because of inadequate primary repair after delivery (Sultan et al, 1994). Surgery, usually lateral sphincterotomy, aimed at relieving an anal fissure by dividing the lower end of the anal sphincter, or haemorrhoidectomy, can inadvertently damage the anal sphincter, causing either passive seepage and/or frank urge incontinence

Sphincter damage can also be caused by other injuries to the perineum or by unwanted sexual penetration of the anal canal (Engel et al, 1995).

Congenital conditions such as imperforate anus can lead to sphincter disruption. Muscle degeneration also can occur for no identifiable reason (Vaizey et al, 1997), and some individuals have weak or disrupted sphincters for which no cause can be established.

\section{Non-sphincter causes}

Diarrhoea or intestinal hurry with frequent and loose bowel actions may indicate under-lying bowel disease such as Crohn's disease or ulcerative colitis, especially if the stool contains blood and mucus. Faecal incontinence may result from the generation of excessively high pressures by the bowel, which even a normal sphincter will at times have difficulty in opposing (Herbst et al, 1997).

Irritable bowel syndrome, often characterized by patterns of frequent loose motions alternating with constipation, may also cause episodes of incontinence, especially if the anal sphincter has been previously impaired by childbirth.

A major surgical resection of the bowel can lead to a loose, unpredictable bowel habit, as can pelvic radiotherapy. Rectal prolapse or an anal or recto-vaginal fistula can also cause leakage of liquid stool or mucus. This is usually rectified by surgery, but for some individuals bowel control remains compromised even after surgical repair.

Neurological disease such as spinal cord injury, multiple sclerosis, and spina bifida may also cause difficulties with effective defecation or continence, both of which require complex neuromuscular coordination.

In immobile people or those prone to severe constipation, passive leakage of liquid stool can occur secondarily to an overloaded colon. This usually ceases after bowel evacuation. The terms 'overflow' and 'spurious diarrhoea' are frequently used to describe this, and it is still unclear why it occurs in some, but not all, people with impaction (Barrett, 1993).

Individuals with deterioration in cognitive function, as seen in dementia, may suffer from incontinence or impaction with overflow due to a combination of environmental, cognitive and neurological factors. 
Anyone with a physical disability may fail to achieve adequate toilet access and become incontinent because of a poorly adapted environment rather than a malfunctioning bowel. Where formal or informal carers are involved in the individual's personal care, their attitudes and practices may fail to facilitate bowel continence.

\section{Assessment}

Individuals with faecal incontinence frequently struggle to cope with this symptom. They feel embarrassed and ashamed, likening themselves to babies who have no control over their bowel actions. They are also uncertain as to what help they can access and seem to feel that, as incontinence is not an illness, there is 'nothing that can be done to help me'. Because of the stigmatizing nature of faecal incontinence, and the effect on quality of life, individuals will not necessarily volunteer this information automatically.

Community nurses have many opportunities to become aware of possible problems with bowel control:

- During a holistic assessment at first visit by a district nurse

- As part of disease management by the practice nurse

- During any continence assessment for bladder problems

- During a health visitor's consultation to assess maternal health.

Many people find it very difficult to talk publicly about their bowels, and often do not have a vocabulary of words they consider socially acceptable to describe what is happening. However, supportive and gentle enquiry, with appropriate prompts and reassurance, can encourage people to voice initial questions and concerns that can open the way for further discussion and treatment.

Investigation must include a detailed history of bowel function, symptoms and the effect on lifestyle. An attempt should be made to gauge whether any psychological processes have precipitated or perpetuated the symptoms.

Formulation of an assessment sheet of relevant questions provides a framework from which further action can be taken. If a nurse suspects that the patient has faecal incontinence, it is important that questioning should be empathic and facilitate discussion, and that an outcome be agreed as to what action the nurse will then take. Otherwise, patients will feel increasingly hopeless if they have divulged this information and no further action is taken. An assessment may indicate to the nurse the cause of the patient's incontinence, for which he/she can give advice and provide treatment.

\section{Assessment sheet}

An assessment sheet (Table 1) can form part of care planning if faecal incontinence is suspected or needs to be identified following anal or abdominal surgery, radiotherapy, a spinal injury, or as part of antenatal and post- natal care. Exploring responses to the following questions will provide the nurse with a history of the patient's bowel function and identify any changes that may require further investigation and treatment.

Usual bowel pattern: 'How often do you open your bowels?' 'Normal' bowel function varies between three times a day to once in 3 days (Connell et al, 1965). It is probably only a minority of 
the population who have their bowels open once each day (Heaton et al, 1992). It is important to record any report of a recent change in the frequency of bowel motions as this may indicate underlying disease or malignancy. Any recent unexplained change in bowel habit in a patient over 40 years of age should be investigated by barium enema X-ray or colonoscopy.

Usual stool consistency: 'What is your stool like: is it loose, soft but formed, hard or hard pellets? Does this vary?' It is important to record any report of an alteration in stool consistency or change in stool colour. Patients with irritable bowel syndrome are particularly prone to a variable stool consistency. Where the patient has difficulty in describing the stool, a visual prompt may be helpful (Figure 2). Type 3 or 4 is the most desirable consistency. Hard pellet stool can often be improved by the addition of fibre to the diet or as a supplement. Very loose stool can also sometimes be firmed by fibre to 'mop up' excess fluid, or by antidiarrhoeal medication such as loperamide.

Presence of blood and/or mucus: 'Do you pass any blood or mucus when you have your bowels open?' Fresh blood may be passed if a patient has haemorrhoids or an anal fissure. Darker blood may indicate underlying disease or malignancy. Passing blood and mucus in an individual with a history of straining may indicate a solitary rectal ulcer. Copious mucus with normal bowel function may indicate a villous adenoma. Disturbed bowel function and the passage of mucus are frequent symptoms of irritable bowel syndrome. A report of passing blood and/or mucus warrants further investigation.

Pain: 'Do you have pain associated with opening your bowels? Is this before opening your bowels and relieved by opening your bowels, or is it a pain as you actually pass a stool?' Pain with an urge to defecate may be reported by patients with inflammatory bowel disease or irritable bowel syndrome, making it difficult to defer defecation. They may refer to a colicky or spasmodic 'stabbing' pain. Pain with defecation is often caused by haemorrhoids or an anal fissure.

Urgency and ability to defer defecation: 'When you need to open your bowels, do you need to rush to get to the toilet? How long can you hold on for?' Patients generating high bowel pressures, as occur in inflammatory bowel disease and irritable bowel syndrome, may report urgency.

Defecation can normally be deferred for long periods of time as the urge to defecate is opposed by voluntary contraction of the external anal sphincter (Whitehead et al, 1981), which should generate sufficient pressure to prevent immediate stool expulsion and to return the stool to the rectum (Figure 3a and $b$ ). If the external sphincter is not functioning well, the squeeze may be insufficient to suppress the urge to defecate, even when the stool is a normal consistency. Patients suffering from diarrhoea find this especially difficult, owing to both increased bowel pressures opposing sphincter function and loose stools.

Urge incontinence: 'Do you ever not get to the toilet in time and have a bowel accident?' This often highlights a weakness or defect in the external anal sphincter, which is unable to oppose rectal contractions and so allow delay in defecation. Urgency may become a persistent problem for an individual who has suffered an episode of faecal incontinence, as the catastrophic effect leads to the fear of future accidents and an immediate response to the urge to defecate to try to prevent this.

It is important to establish how frequently urge incontinence actually occurs and whether restrictions in activities are due to actual or feared incontinence. Some people have very infrequent 
episodes, but self- impose major lifestyle restrictions and never venture far from a toilet 'just in case'.

Evacuation difficulties: 'Do you have difficulty in opening your bowels? Do you need to strain? If so, for how long? Do you ever need to insert a finger into your back pa sage to help stool out? Do you need to push on the area by your back passage? Does it feel as if you have not completely emptied your bowels?' These questions examine whether the patient suffers from an evacuation difficulty. Patients with weak sphincters often feel unable to propel the stool out of the anal canal or they may have loose stools which are incompletely evacuated and therefore cause passive soiling. Some women develop a rectocele following child- birth or excessive straining. The front wall of the rectum may bulge into the vagina with attempted defecation. Sometimes gentle backward pressure with a finger in the vagina may aid complete evacuation of any stool 'trapped' in a rectocele.

Passive soiling: 'Do you have any leakage from your back passage, of which you are unaware? Is this liquid or solid? Does this occur at any time or only after you have opened your bowels?' The internal anal sphincter is responsible for resting tone in the anal canal. A weak or disrupted sphincter will not close the anal canal completely; therefore, if the stool is loose or soft, some will remain in the anal canal and will ooze out after defecation. These patients complain of great difficulty in cleaning the anus after defecation and subsequent soiling, possibly for several hours. Patients may be helped by taking loperamide tablets or syrup to firm the stool.

Flatus: 'Can you control your wind? Are you able to tell the difference between wind and the need to empty your bowels?' These questions also examine the function of the sphincters and whether they are able to maintain an airtight seal. Some patients report incontinence of liquid or solid stool on passing flatus, because they are unable to distinguish between flatus and stool.

Incontinence of flatus is difficult to treat. Dietary changes, such as reduction in bran products, vegetables and caffeine, can reduce the amount of flatus produced for some people. Products such as live natural yoghurt and aloe vera are reported as helpful in reducing flatus by some patients (Norton and Kamm, 1999).

Pads or pants: 'Do you need to wear a pad because of problems with leakage from your bowels? If so, what type of pad ? Do you need to change your underwear during the day due to leakage? If yes, how often?' Patients may wear pads for urinary incontinence and so it is important to establish whether pads or underwear are changed because of faecal incontinence. Also, patients who describe urgency may always wear a pad, because of fear of incontinence rather than actual accidents

Obstetric history: 'How many babies have you had? Were forceps used for any of these deliveries? Did you tear, or did you have stitches? How heavy were the babies? Was there any problem with bowel control following the deliveries?' As indicated earlier, tears, prolonged labour (particularly the second stage), and heavy babies can all cause trauma and damage to the anal sphincters. Although, postnatally, women are frequently asked about bladder function and taught the importance of pelvic floor exercises, bowel function is much less frequently considered or mentioned. Women frequently report feeling embarrassed to raise this issue themselves. 
Bladder control: 'Do you have any problems with leakage from your bladder? Does urine leakage occur if you cough, sneeze or laugh? Do you need to rush to the toilet to pass water?' These questions help to identify any other continence symptoms. In women, these problems may indicate a weak pelvic floor. Referral for further investigation may be indicated.

\section{Effect on lifestyle/ relationships/psychological factors.}

If a patient reports symptoms of incontinence it is important to ascertain how this has affected his/her lifestyle. Some patients report feeling very restricted, planning their journeys around toilet facilities, while others may become house-bound. Such behaviour will obviously have a profound effect on the individual, and any partner and family. Patients often report avoiding a sexual relationship because of feeling dirty or a fear that an episode of incontinence will occur.

\section{Investigations}

As well as a detailed history, patients should have a digital rectal examination to check for local pathology. Patients with a recent unexplained change in bowel habit need at minimum a sigmoidoscopy, and preferably a barium enema or colonoscopy. Where the cause of impaired bowel control is unclear, or first-line management has failed, the patient will need further investigations.

Ano-rectal physiology tests and anal ultrasound are used to assess the function and structure of the internal and external anal sphincters. Ano-rectal physiology tests also assess the sensitivity and neurological function of the rectum, using balloon distension and electrical stimulation. Any disruption of the anal sphincters can be clearly seen on the anal ultrasound. These tests take about 1 hour and are available in many centres around the country. Any patient with persistent, poor bowel control should have access to them.

\section{Conclusion}

Assessment of bowel control requires appropriate sensitive questioning that will facilitate discussion and allow patients to voice any symp toms or anxieties. Nurses often have access to people at risk of faecal incontinence and it is important that they use every opportunity to allow discussion of this sensitive issue.

Patients may express a sense of relief at being asked about their bowel control and being given the opportunity to ask questions and seek help, as unfortunately 'bowels' remain a taboo subject.

Treatments are available for most of the problems identified (Norton, 1997; Kamm, 1998; Norton and Kamm, 1999).

Active publicity for bowel control problems is also starting to appear and it is hoped that in the future increasing numbers of people will come forward for help.

\section{References}

Barrett JA (1993) Faecal Incontinence and Related Problems in the Older Adult. Edward Arnold, London 
Connell AM, Hilton C, Irvine G, Lennard-Jones JE, Misiewicz JJ (1965) Variation in bowel habit in two population samples. Br Med J 2 :1095-9

Engel AF, Kamm MA, Bartram Cl (1995) Unwanted anal penetration as a physical cause of faecal incontinence. Eur J Gastroenterol Hepatol 7: 65-7

Heaton KW, Radvan J, Cripps H, Mountford RA, Braddon FEM, Hughes AO (1992) Defecation

frequency and timing, and stool form in the general population: a prospective study. Gut 33: 818-24

Herbst F, Kamm MA, Morris GP, Britton K, Woloszko J, Nicholls RJ (1997) Gastrointestinal transit and prolonged ambulatory colonic motility in health and faecal incontinence. Gut 41:381-9

Johanson JF, Lafferty J (1996) Epidemiology of fecal incontinence: the silent affliction. Am J

Gastroenterol 91:33-6

Kamm MA (1998) Faecal incontinence: clinical review. Br Med J 316:528-32

Leigh RJ, Turnberg LA (1982) Faecal incontinence: the unvoiced symptom. Lancet i:1349-51

Nelson R, Norton N, Cautley E, Furner S (1995) Community-based prevalence of anal incontinence. JAMA 274: 559-61

Norton C (1997) Faecal incontinence in adults 2: treatment and management. Br J Nurs 6 (1): 23-6

Norton C, Kamm MA (1999) Bowel Control: Information and Practical Advice. Beaconsfield

Publishers, Beaconsfield (In press)

Royal College of Physicians (1995) Incontinence:causes, management and provision of services. A working party of the Royal College of Physicians. J R Coll Physicians Lond 29: 272-4

Sultan AH, Kamm MA, Hudson CN, Bartram Cl (1994) Third degree obstetric and sphincter tears: risk factors and outcome of primary repair. Br Med J 308: 887-91

Sultan AH, Kamm MA, Hudson CN, Thomas JM, Bartram Cl (1993) Anal sphincter disruption during vaginal delivery. N Engl J Med 329: 1905-11

Vaizey CJ, Kamm MA, Bartram Cl (1997) Primary degeneration of the internal anal sphincter as a cause of passive faecal incontinence. Lancet 349: 612-15

Whitehead WE, Orr WC, Engel BT, Schuster MM (1981) External anal sphincter response to rectal distension: learned response or reflex. Psychophysiology 19: 57-62 
Table 1. Questions to include in assessment

- Usual bowel pattern Usual stool consistency Blood and/or mucus?

- Pain prior to or with defecation

- Urgency and ability to defer defecation

- Urge incontinence Evacuation difficulties Passive soiling

- Ability to control flatus and/or distinguish from stool

- Pads/pants Obstetric history Bladder control

- Effects on lifestyle, relationships and psychological factors 